\title{
Are Basophils and T helper 2 Cells Implicated in Mastocytosis?
}

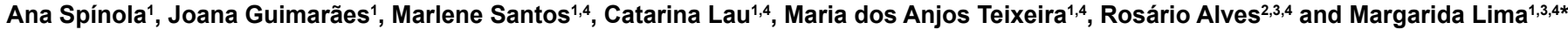 \\ ${ }^{1}$ Laboratory of Cytometry, Department of Hematology, Hospital de Santo António (HSA), Centro Hospitalar do Porto (CHP), Porto, Portugal \\ ${ }^{2}$ Department of Dermatology, Hospital de Santo António (HSA), Centro Hospitalar do Porto (CHP), Porto, Portugal \\ ${ }^{3}$ Mutidisciplinar Consultation for Cutaneous Lymphomas and Mastocytosis, Hospital de Santo António (HSA), Centro Hospitalar do Porto (CHP), Porto, Portugal \\ ${ }^{4}$ Unit for Multidisciplinary Investigation in Biomedicine (UMIB), Instituto de Ciências Biomédicas Abel Salazar (ICBAS), Universidade do Porto (UP), Porto, Portugal
}

\begin{abstract}
Background: Clinical signs and symptoms observed in patients with mastocytosis are not only related to organ infiltration, but also result of either local or systemic mast cells mediator release. Knowing that mast cells, basophils and T helper 2 (Th2) cells have been implicated in allergy, we hypothesized that basophils and Th2 cells could also be involved in the episodes of mediator release often seen in patients with mastocytosis and, in a small proportion, could be on the bloodstream in an activated mode and/or be over-reactive to stimuli.

Methods: We aimed to quantify and characterize, by flow cytometry, the basophil (CD45+dim, CD123+bright, CD294/CRTH2+, CD3- and HLA-DR-), Th2 (CD3+, CD294/CRTH2+) and activated (CD3+, HLA-DR+) T cell populations in the peripheral blood from 19 patients with mastocytosis, as well as the ability of peripheral blood basophils to be activated, thereby expressing CD63 and down-regulating CD193/CCR3 expression on the cell surface, in response to different stimuli (fMLP and anti-Fc2RI), as compared with 19 normal individuals.
\end{abstract}

Results: We found no evidence for increased basophil, Th2 and activated T cell counts, neither for over-reactive basophils in response to fMLP and anti-Fc2RI, in mastocytosis patients, as compared with normal individuals.

Conclusions: Using the present methodology, our results argue against a possible role of basophils and Th2 cells in the episodes of mediator release observed in patients with mastocytosis.

Keywords: Mastocytosis; Allergy; Basophils; Th2 cells

Abbreviations: BAT: Basophil Activation Test; CRTH2: G proteincoupled Receptor-44 or Chemoattractant Receptor Expressed on Th2 cells; EDTA-K3: Ethylene-Diamine-Tetracetic Acid; ENPP-3: Ectoenzyme ectonucleotide Pyrophosphatase/Phosphodiesterases 3; IL: Interleukin; ISM: Indolent Systemic Mastocytosis; LAMP: Lysosomal Membrane Proteins; MFI: Median Fluorescence Intensity; PGD2: Prostaglandin D2; PI3K: Phosphatidylinositide 3-Kinases; SM: Systemic Mastocytosis; Th2: T helper 2; WHO: World Health Organization

\section{Introduction}

Mast cells reside in tissues, generally located near epithelial surfaces, blood vessels, nerves and glands. They take part in many modes of immune response, including innate and adaptive immune responses [1]. Upon stimulation, they de-granulate, release and synthesize proinflammatory, vaso-dilative, chemotactic and cytotoxic mediators.

Mastocytosis are rare clonal hematological disorders characterized by neoplastic proliferation and accumulation of mast cells in one or more organs or tissues [2-4]. They are believed to be caused by acquired somatic mutations in critical receptors and intracellular signaling pathways that control mast cell proliferation and survival. It may occur at any age, even be present at birth; the male to female ratio reported vary from $1: 1$ to $1: 3$ with a slight male predominance in cutaneous mastocytosis. Several mastocytosis subtypes were recognized in the World Health classification (WHO), last updated in 2008 [5]. From them, cutaneous mastocitoma is the most frequent variant observed in children, whereas Indolent Systemic Mastocytosis (ISM), often manifesting in the skin as urticaria pigmentosa, is the most frequently diagnosis in adults [4]. Clinical signs and symptoms observed in patients with mastocytosis are not only related to organ infiltration, but also result of either local or systemic mast cells mediator release [6]. They include skin lesions (pruritus, urticaria, dermatographism), mediator-related events (flushing, hypotension, syncope, palpitations, tachycardia, headache, abdominal pain, diarrhea or even anaphylaxis) and nonspecific constitutional symptoms (fatigue, weight loss, anorexia, fever and diaphoresis). These manifestations vary from mild to severe or even life threatening and can occur spontaneously or in response to stimuli. A variety of triggers can generate activation of mast cells such as heat, cold, pressure, alcohol, food, medications, radio contrast agents and venoms; response to these stimuli cannot be predictable and diverse between patients.

Although mast cells are often considered the primary effector cell in allergy [7], previous studies have been shown that basophils are also a main component of both immediate allergy and inflammation that frequently accompanies chronic allergic reactions [1,8]. Indeed, although there is still some controversial points, several approaches indicate that basophils can migrate into inflamed tissues where they cooperate with other immune cells to promote optimal $\mathrm{T}$ helper 2 (Th2) cytokine-mediated responses, and, in some circumstances, contributing to allergy [8]. Immuno-phenotypically, basophils are known to express CD9, CD13, CD22, CD25 (dim), CD33, CD36, CD38 (bright), CD45 (dim), CD123 (bright), CD193 (CCR3), CD294 and FceRI, the high-affinity IgE receptor, and lack expression of CD15,

*Corresponding author: Margarida Lima, Laboratory of Cytometry, Department of Hematology, Hospital de Santo António, Centro Hospitalar do Porto, Rua D. Manuel II, s/n, 4099-001 Porto, Portugal, Tel: 351226004808; E-mail: margaridalima@chporto.min-saude.pt

Received August 14, 2014; Accepted September 22, 2014; Published September 29, 2014

Citation: Spínola A, Guimarães J, Santos M, Lau C, Teixeira MA, et al. (2014) Are Basophils and T helper 2 Cells Implicated in Mastocytosis? J Allergy Ther 5: 192. doi:10.4172/2155-6121.1000192

Copyright: $\odot 2014$ Spínola A, et al. This is an open-access article distributed under the terms of the Creative Commons Attribution License, which permits unrestricted use, distribution, and reproduction in any medium, provided the original author and source are credited. 
CD 117 and HLA-DR [9,10]. Among these markers, CD294, also known as CRTH2 (G protein-coupled receptor-44 or chemoattractant receptor expressed on Th2 cells), a major receptor for Prostaglandin D2 (PGD2), a prostanoid produced mainly by mast cells that has been shown to induce vasodilatation and increase permeability, as well as to promote leukocyte chemotaxis during allergic responses $[11,12]$.

When activated, basophils are identified by flow cytometry based on the expression of surface markers of degranulation [6]. CD63 is a tetra-spanin molecule present in the lysosomal and secretory granule membranes that is not expressed at the plasma membrane of resting basophils, but reaches the plasma membrane as result of granule translocation and membrane fusion [13]. Other cell surface antigens that have been described as activation-linked markers include the amino-peptidase $\mathrm{N}$ (CD13), the ecto-enzyme ecto-nucleotide pyrophosphatase/phosphor-di-esterase 3, ENPP-3 (CD203c), the lysosomal membrane proteins LAMP-1 (CD107a), LAMP-2 (CD107b), and LAMP-3 (CD63), and endolyn (CD164) [14-16]; these molecules are detectable at low levels on the surface of resting blood basophils, their expression increasing after basophil activation due to translocation from cytoplasmic (lysosomal) membranes onto the cell surface. Plasma membrane CD203c undergoes very rapid up-regulation after basophil activation and mirrors early activation processes which may not involve complete basophil degranulation [15]. Basophil activation also results in a continuous increase of the CD203c expression, limits between non-activated (CD203c dim) and activated (CD203c bright) basophils being somewhat subjective, while CD63 expression is an allor-nothing phenomenon [14]. In contrast, CD193 (CCR3) expression often decreases during basophil activation, as CCR3 molecules are dispersed in the plasma membrane of resting basophils and they aggregate in lipid rafts during basophil activation [17].

At least two functional subsets of $\mathrm{T}$ cells with distinct cytokine secretion profiles have been described: Th1 cells, which produce predominantly interferon-gamma and interleukin (IL)-2 and are involved in cell mediated immune responses, and Th2 cells, which secrete IL-4, IL-5, IL-10 and IL-13, participate in IgE-mediated immune responses and are involved in allergy [18]. As basophils and eosinophils, Th2 cells have been shown to express the CD294 (CRTH2) molecule [19-21], which determines the recruitment of basophils, eosinophils and Th2 cells to the allergic inflamed tissues by interacting with PGD2 [11] and suppresses the apoptosis of Th2 cells by activating the PI3K (Phosphatidylinositide 3-Kinases) pathway [22,23].

Knowing that mast cells are implicated in allergy and that basophils share similar functions, we hypothesized that basophils and Th2 cells may be skewed to an allergic phenotype by the mast cell dominant cellular environment in mastocytosis and that basophils and Th2 cells could be involved in the episodes of mediator release that often seen in patients with mastocytosis and, in a small proportion, could be on the bloodstream in an activated mode and/or be over-reactive to stimulus. Therefore, we aimed to quantify and characterize, by flow cytometry, the basophil and Th2 cell populations in the peripheral blood from patients with mastocytosis, as compared with normal individuals without history of allergy. We also intended to look for possible correlations between the basophil activation status and the episodes of mediator release observed in patients with mastocytosis.

\section{Material and Methods}

\section{Patients and controls}

Nineteen adult patients with mastocytosis were included, and they were classified accordingly to the according to the World Health Organization classification, 2008 [5]. In addition, 19 gender and agematched normal individuals (blood donors) with no history of allergies were used as controls. To the patients, if applicable, it was requested to stop sodium cromoglycate and anti-histamines 1 day before the blood sample collection and to return the usual dosage after it. None of the patients was medicated with corticosteroids.

\section{Biological samples}

Patients and donors collected $3 \mathrm{~mL}$ of tripotassium ethylenediamine-tetracetic acid (EDTA-K3) whole-blood samples. All tests were carried out within 6 hours after blood sampling.

\section{Ethical statement}

The institutional scientific and ethic committees approved this study. All patients and controls provided informed consent.

\section{Blood cell counts}

The leukocyte differential count was performed using a LH780 hematology analyzer (Beckman-Coulter).

\section{Flow cytometry}

Basophil quantification and phenotypic characterization: Cell surface immunophenotyping was performed using a whole blood stainlyse-and-then-wash 6-color direct immunofluorescence technique. Briefly, after adjusting the cell concentration to $5-15 \times 10^{6}$ cells $/ \mu \mathrm{l}, 100$ $\mu \mathrm{l}$ of whole blood were incubated for 15 minutes at room temperature with the appropriate volume of fluorochrome conjugated monoclonal antibodies directed against relevant antigens (Table 1), followed by erythrocyte lysis and cell fixation, using FACS lysing solution ${ }^{\circledR}$, from Becton-Dickinson Biosciences, San José, California, USA (BDB), according to the manufacturer's instructions.

Basophil response to IgE and non-IgE mediated reactions: Basophil response to IgE and non-IgE mediated reactions was evaluated using Flow CAST $^{\mathbb{B}}$ Basophil Activation Test (BAT) (Bühlmann Laboratories $\mathrm{AG}^{\mathbb{}}$, Schönenbuch, Switzerland), by measuring the CD63 expression at the basophil surface [24]. Samples were processed according to the manufacturer's instructions. Briefly, stimulation buffer containing calcium, heparin and IL-3 (for basophil priming) was added to whole blood samples and anti-FceRI or the unspecific cell activator fMLP (formyl-Methionyl-Leucyl-Phenylalanine) were used to activate basophils. Staining reagent containing a mixture of $\mathrm{mAb}$ to the chemokine receptor CCR3 (CD193) labeled with phycoerythrin, and to CD63 labeled with fluorescein isothiocyanate, where used to stain basophils and to detect basophil activation, respectively. Erythrocytes were removed by a lysing reaction and after a short centrifugation step the cells were suspended in wash buffer and analyzed by flow cytometry.

\begin{tabular}{|l|c|c|c|c|}
\hline Specificity & Clone & Isotype & Manufacturer & Fluorochrome \\
\hline HLA-DR & L234 & $\operatorname{lgG} 2 a$ & BioLegend & PB \\
\hline CD45 & HI30 & $\operatorname{lgG1}$ & Invitrogen & PO \\
\hline CD294 (CRTH2) & BM16 & $\operatorname{lgG} 2 a$ & BioLegend & PE \\
\hline CD123 (IL-3R) & $6 H 6$ & $\operatorname{lgG1}$ & BioLegend & PE-Cy7 \\
\hline CD3 & SK7 & IgG1 & BDB & APC \\
\hline
\end{tabular}

Abbreviations: APC: Allophycocyanin; PE: Phycoerythrin; PB: Pacific Blue PO: Pacific Orange; IL-3R: Interleukin 3 Receptor; CRTH2: G protein-coupled receptor-44 or chemoattractant receptor expressed on Th2 cells.

Manufacturer: BioLegend ${ }^{\circledR}$, San Diego, CA; Invitrogen ${ }^{\circledR}$, Camarillo, CA; Becton Dickinson Biosciences (BDB), San Jose, CA

Table 1: Clones and specificities of the monoclonal antibodies used in this study. 
Un-stimulated samples were used as negative controls.

Flow cytometry acquisition: Data acquisition was performed in a FACS Canto v.2 flow cytometer (Becton Dickinson-BD, San Jose, CA, USA), using the FACSDiva software (BD). A minimum of 200,000 events was acquired in order to acquire at least 1,000 basophils in each sample.

Flow cytometry data analysis: The Infinicyt ${ }^{\mathrm{TM}}$ (Cytognos, Salamanca, Spain) program was used for data analysis. Briefly, low sidescattered (SSC), CD45+dim, CD123+bright, CD294 (CRTH2)+, CD3and HLA-DR-cells were identified as basophils and the percentage of basophils among white blood cells was calculated. The use of SSC/ CD45 dot plot contributed to discriminate basophils area from other leukocytes better than FSC/SSC light-scattering, excluding cellular debris and to account for a further selective marker to phenotype basophilic cells based on CD123 and CD294 expression [10] (Figure 1, panel A). Afterwards, basophils were characterized for the expression of the cell surface antigens analyzed, using both the percentage of positive cells and the median fluorescence intensity (MFI) of antigen expression. In addition, the percentage of $\mathrm{T}(\mathrm{CD} 3+)$ cells within lymphocytes and the fraction of HLA-DR+ T cells, as well as CD294(CRTH2)+ HLA$\mathrm{DR}+\mathrm{T}$ cells were quantified in each individual sample (Figure 1, Panel B).

\section{Statistics analysis}

Statistical analysis was performed using the IBM SPSS Statistics 20 software. For all continuous quantitative variables, the median, mean, standard deviation and range values were calculated. The correlation between basophil count in automatic hematological counter and flow cytometry was evaluated using the Spearman's coefficient test. The Mann-Whitney test was used to compare continuous quantitative variables, such as the $\%$ of positive cells and the MFI for the markers analyzed. P value less than 0.05 was considered statistically significant.

\section{Results}

\section{Clinical characteristics}

Nineteen patients with the diagnosis of mastocytosis were studied, 7 men and 12 women, with a median age of 47 years, ranging from 17 to 83 years. The median age of disease onset was 35 years, ranging from 0.67 to 62 years.

By the moment of the diagnosis, 17 patients (89\%) had more than 5 years evolution since the first skin lesions, in 11 patients the lesions got worse and in 5 patients the lesions did not change with time.

Regarding to the classification of mastocytosis, the population consisted of 9 patients with ISM, 1 with systemic mastocytosis with an associated hematologic non-mast cell lineage disorder (SM-AHNMD) and 4 with CM. In the remaining 5 cases, it was not possible to establish the differential diagnosis between ISM and CM, since the bone marrow investigation was not performed.

The main clinical and laboratory findings are summarized in Table 2. All patients had skin lesions consistent with the diagnosis of urticaria pigmentosa. Even not having a preferential location the lesions were more frequently seen in the trunk. Other areas such as abdomen, arms and legs were also involved, having the majority of the patients $(\mathrm{n}=12)$ more than one area affected. At the diagnosis, 2 cases presented with disseminated cutaneous involvement.

Fourteen patients had pruritus, usually moderate and typically described as itching of the lesions, 8 of them reported to have an additional symptom, which was a burning sensation at the lesions in 5 cases and needle sting feeling in another 3 cases. Other symptoms were: gastrointestinal in $84 \%(n=16)$, osteoarticular in $74 \%(n=14)$, allergic
A A1

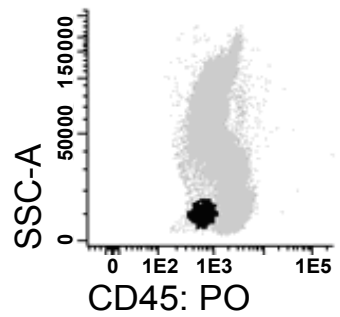

B

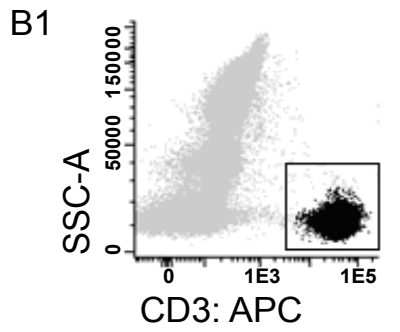

A2

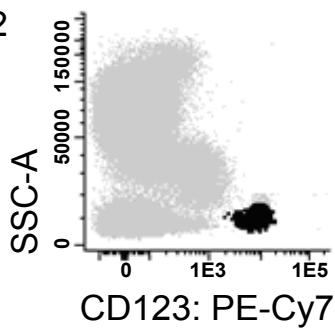

B2

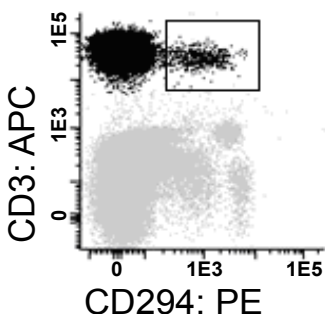

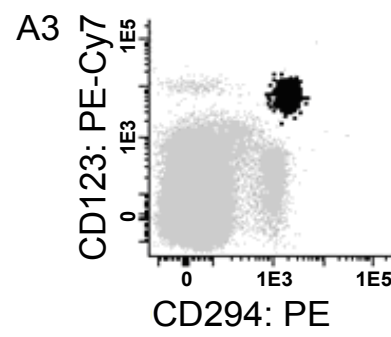

B3

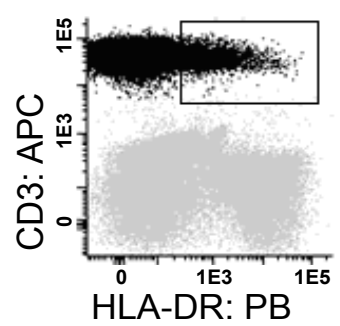

Figure 1: Dot plots illustrating the gating strategy used for the immunophenotypic identification and characterization of basophils (Panel A) and T cells (Panel B) in peripheral blood samples. Panel A: Basophils (black events) were selected as SSC (low) CD45+dim (A1) and CD123+bright (A2) events and analyzed for the expression of CD123 and CD294 (A3). Panel B: T cells (black events) were selected as SSC (low), CD3+ events (B1) and analyzed for the expression of CD294 (CRTH2) (B2) and HLA-DR (B3). Other peripheral blood leukocytes are shown as grey events. 


\begin{tabular}{|c|c|}
\hline Gender (males: females) & $7: 12$ \\
\hline Age (years old) & $47[17-83]$ \\
\hline \multicolumn{2}{|l|}{ Presenting features } \\
\hline Age at onset (years) ${ }^{\star}$ & $35[0.67-62]$ \\
\hline Age at diagnosis (years) ${ }^{\dagger}$ & $44[15-70]$ \\
\hline Time between the onset and diagnosis (years) & $16[3-48]$ \\
\hline Follow-up from diagnosis (years) ${ }^{\S}$ & $3[0.6-18]$ \\
\hline \multicolumn{2}{|l|}{ Disease manifestations } \\
\hline Pruritus & $14(74 \%)$ \\
\hline Cutaneous & $19(100 \%)$ \\
\hline Gastrointestinal & $16(84 \%)$ \\
\hline Osteoarticular & $14(74 \%)$ \\
\hline Neuropsychiatric & $9(47 \%)$ \\
\hline Allergic & $10(53 \%)$ \\
\hline Cardiovascular & $9(47 \%)$ \\
\hline \multicolumn{2}{|l|}{ Skin lesions } \\
\hline Generalized & $2(11 \%)$ \\
\hline Restricted to one area & $6(32 \%)$ \\
\hline More than one area involved, but not generalized & $11(58 \%)$ \\
\hline \multicolumn{2}{|l|}{ Laboratory findings at presentation } \\
\hline Total $\lg E(\mathrm{KU} / \mathrm{L})^{\ddagger}$ & $10.3[<2-231]$ \\
\hline Tryptase $(\mathrm{g} / \mathrm{L})^{\S}$ & $24.9[4-305]$ \\
\hline
\end{tabular}

Results are expressed as median [range] values, absolute numbers and (percentages).

Date of the first mast cell mediators release episode or appearance of skin lesions tDate of the first bone marrow study or skin biopsy

$\ddagger$ Normal values: $\lg \mathrm{E}<100 \mathrm{KU} / \mathrm{L}$

\$Normal values: Tryptase $<13.5 \mu \mathrm{g} / \mathrm{L}$

Table 2: Clinical and laboratory characteristics from the patients included in this study.

in $53 \%(\mathrm{n}=10)$, neuropsychiatric in $47 \%(\mathrm{n}=9)$ and cardiovascular in $47 \%(n=9)$. Allergic manifestations included allergic reaction to insect bites ( 5 cases), allergic rhinitis ( 4 cases), and allergy to medications, bronchial asthma and atopic dermatitis (3 cases each).

All patients identified one or more factors that trigger local and/ or systemic mast cell release mediator episodes with 18 patients identifying more than one factor. Symptoms included worsening of the skin lesions, with increased number and/or color modification to a reddish maculopapula, feeling sick, flushing, abdominal pain and diarrhea and/or aphthous ulcers of the oral mucosa. Food $(n=15)$, sun exposure $(n=15)$, stress and/or anxiety $(n=12)$, temperature changes $(n=11)$, strong emotions $(n=5)$, skin traumatisms $(n=4)$, alcoholic beverages $(n=4)$, sleepless nights $(n=3)$, and exercise $(n=1)$ were the aggravating factors listed. Food not tolerated varied from fruit $(n=9)$, spicy food $(n=6)$, milk or milk derivatives $(n=5)$, chocolate $(n=4)$, seafood $(n=3)$, octopods, fish and/or meat ( 1 each). Strawberries $(n=6)$ and tomatoes $(n=3)$ were the fruits more frequently referred. With regard to temperature variations, the worsening of symptoms with heat $(n=15)$ was reported more frequently than with cold $(n=8)$. One patient had history of systemic mast cell release mediators triggered by morphine; this reaction consisted of shortness of breath, generalized edema and feeling of imminent death.

\section{Basophil count}

Basophils in the peripheral blood samples, as determined by flow cytometry, ranged from 0.07 to $1.76 \%$ of the white blood cells (median, $0.39 \%$ ) in patients, as compared to 0.21 to $1.1 \%$ (median, $0.5 \%$ ) observed in controls $(\mathrm{p}>0.05)$. In overall, there was a positive correlation between the basophil count as determined by flow cytometry and that obtained in the automated blood cell counter $(r=0.477 ; \mathrm{p}=0.003)$.

\section{Basophil immunophenotype}

Basophils in all cases had a low SSC and were positive for CD45 (dimmer than lymphocytes), CD123 (bright) and CD294 (bright) (Figure 1, panel A). In addition, the MFI of CD123, CD294 and CD45 expression observed in basophils from patients with mastocytosis did not differ from that observed in controls (Figure 2).

\section{Basophil response to non-IgE and IgE-mediated reactions}

After stimulation with fMLP or with anti-FceRI, an increase in the percentage of CD63+ basophils was observed in normal individuals as in patients, although some "non responders" were found in both cases (Figure 3, panel A). In the literature the existence of "non responder" individuals in basophil activation tests has been reported when using CD63 to evaluate basophil activation [25]. However, it should be noted that cells "non-responding" to fMLP or anti-FceRI (when evaluated using CD63) are fully responsive to the same agonists when evaluated with other activation-related markers, such as CD203c [26], which was not tested in the present study.

Regarding the MFI of CD193 (CCR3) expression on basophils from unstimulated patient's samples, it was higher than in fMLP-stimulated and in anti-FceRI-stimulated samples $(\mathrm{p}=0.017$ and $\mathrm{p}=0.025$, respectively), and similar results were obtained in controls $(\mathrm{p}=0.045$ and $\mathrm{p}=0.009$, respectively). However, the MFI of CD193 (CCR3)

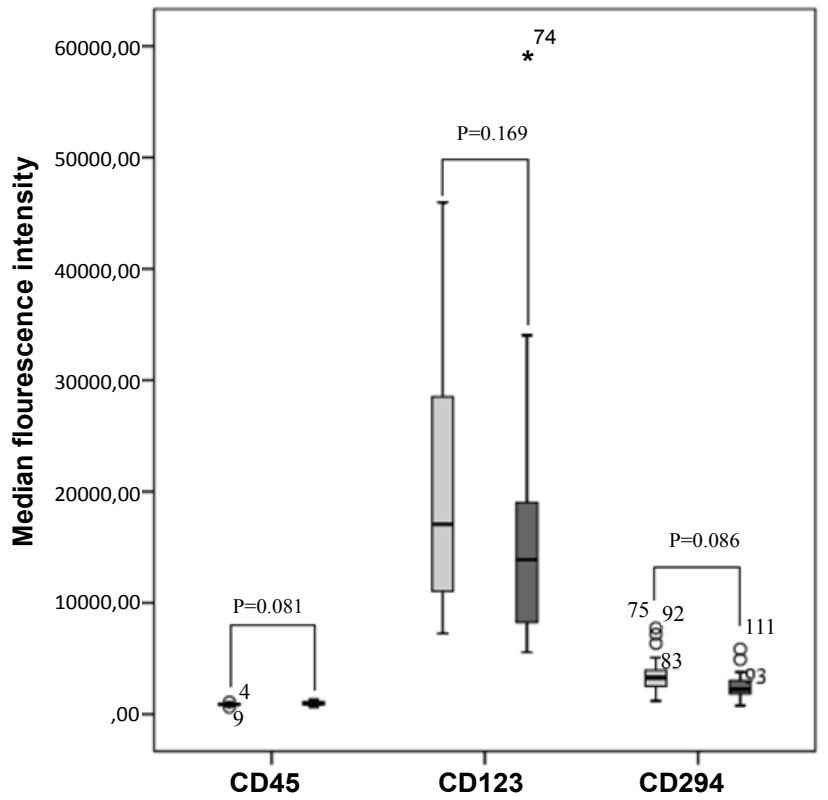

Figure 2: Median fluorescence intensity of CD45, CD123 and CD294 (CRTH2) expression on peripheral blood basophils from patients with mastocytosis ( $n=19$, dark grey boxes) and healthy individuals (blood donors) $(n=18$, light grey boxes)

Pacific orange conjugated anti-CD45, phycoerythrin Cy-7 conjugated antiCD123, and phycoerythrin conjugated anti-CD294 were used in combination with other monoclonal antibodies.

Median fluorescence intensity is expressed as arbitrary units scaled from 0 to 100,000 .

Boxes extend from the $25^{\text {th }}$ to the $75^{\text {th }}$ percentiles; the line in the middle and the vertical lines represent median values and $95 \%$ confidence intervals, respectively. No significant differences $(P>0.05)$ were observed between the median fluorescence intensity of CD45, CD123 and CD294 (CRTH2) expression on peripheral blood basophils from patients and controls using the Mann-Whitney U-test. 
expression between fMLP and anti-FceRI activated basophils did not significantly differ, neither in patients nor in controls ( $p>0.05$ in both cases). Moreover, its expression on basophils did not significantly differ between patients and controls, in unstimulated, as well as in fMLPstimulated and in anti-FceRI-stimulated samples ( $\mathrm{p}>0.05$ in all cases) (Figure 3, panel B).

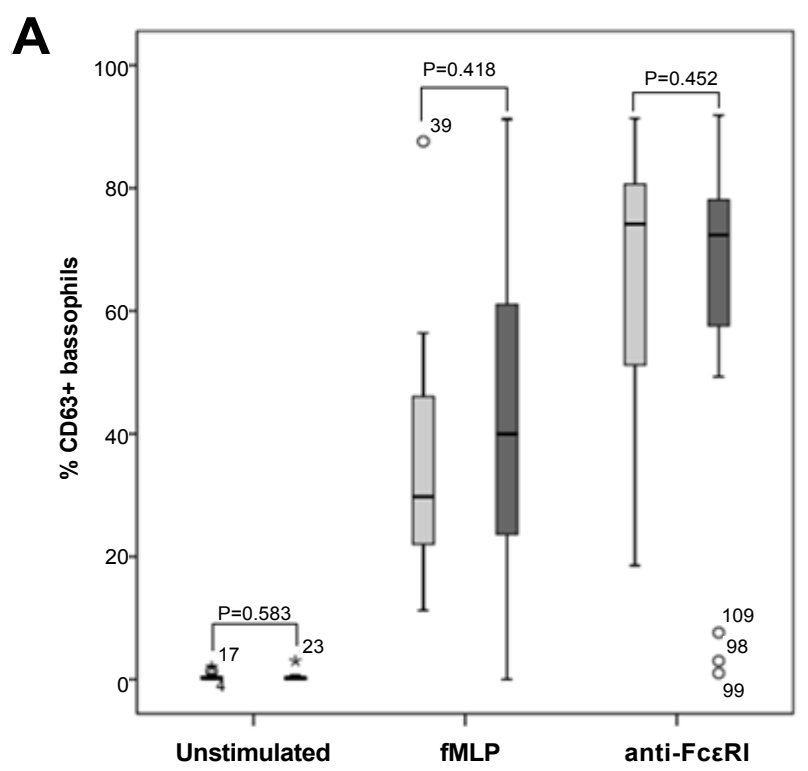

B

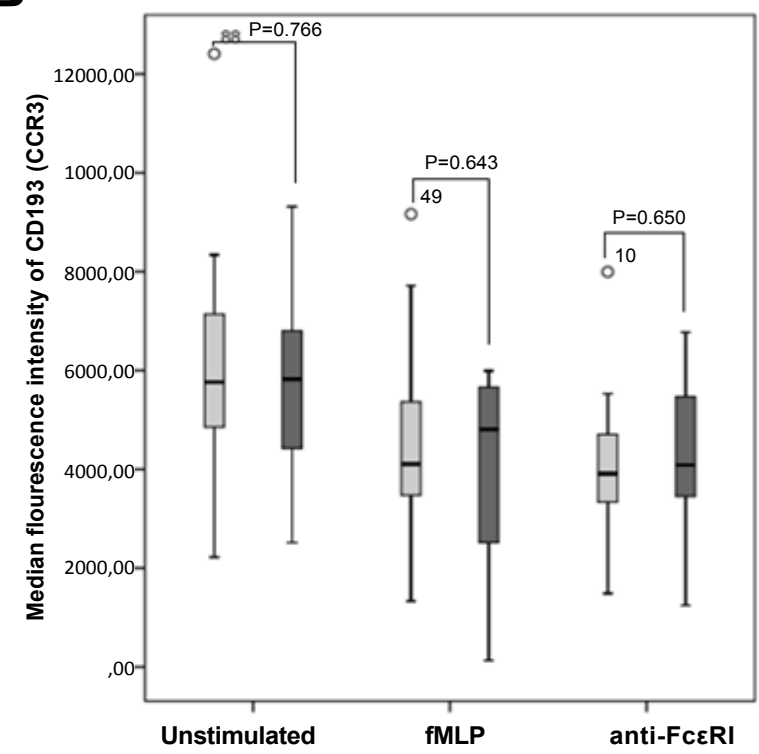

Figure 3: Percentages of CD63 positive activated peripheral blood basophils (Panel A) and median fluorescence intensity of CD193 (CCR3) expression (Panel B) on activated peripheral blood basophils, in patients with mastocytosis $(n=19$, dark grey boxes) and in healthy individuals (blood donors) $(n=19$, light grey boxes), at the baseline (unstimulated) and after stimulation with fMLP or with anti-FceRI.

Boxes extend from the $25^{\text {th }}$ to the $75^{\text {th }}$ percentiles; the line in the middle and the vertical lines represent median values and $95 \%$ confidence intervals, respectively. No significant differences $(P>0.05)$ were observed between the \% of CD63+ basophils and the MFI of CD193 (CCR3) expression on basophils in patients and in controls using the Mann-Whitney U-test.

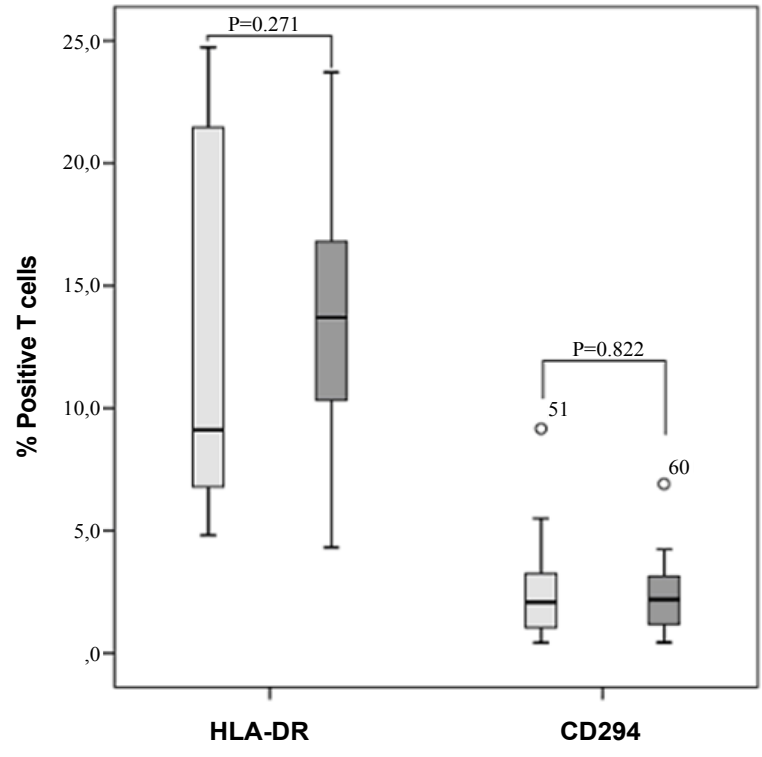

Figure 4: Percentage of HLA-DR+ and CD294 (CRTH2)+ peripheral blood $T$ cells, in patients with mastocytosis ( $n=19$, dark grey boxes) and healthy individuals (blood donors) ( $n=18$, light grey boxes).

Allophycocyanin conjugated anti-CD3, pacific blue conjugated anti-HLA-DR, and phycoerythrin conjugated anti-CD294 were used in combination with other monoclonal antibodies.

Boxes extend from the $25^{\text {th }}$ to the $75^{\text {th }}$ percentiles; the line in the middle and the vertical lines represent median values and $95 \%$ confidence intervals, respectively. No significant differences $(P>0.05)$ were observed between the $\%$ of HLA-DR+ and CD294 (CRTH2)+peripheral blood T cells in patients and controls using the Mann-Whitney U-test.

\section{T cell immunophenotype}

T cells in all cases had a low SSC, and they were positive for CD45 (bright) and CD3 (bright) (Figure 1, panel B). The expression of CD294 (CRTH2) was heterogeneous and the proportion of T cells expressing this molecule was small, median of $2.1 \%$, ranging from 0.4 to $9.0 \%$ (Figure 4) In the same way, HLA-DR, an activation-related marker, was heterogeneously expressed in a small percentage of T cells, ranging from 4.3 to $24.7 \%$ (median $13.2 \%$ ). No significant differences were observed between controls and patients concerning the expression of these markers. Activated Th2 lymphocytes (CD294+HLA-DR+) were not found circulating on higher proportions than in controls $(\mathrm{p}=0.221)$.

\section{Discussion}

Mastocytosis have been associated with diverse allergic reactions to a variety of stimuli. The hypothesis that, patients with this disease, could have activated circulating basophils in a small number and that they were rapidly responders to stimulus theoretically appeared to be reasonable. And it could justify the heterogeneous clinical manifestations observed in these individuals. However, in the present study we could not demonstrate increased basophil counts when compared with healthy controls.

In addition, even with the same trigger, the response could not be predictable by the present of activated circulating basophils at rest stage, as it was shown that, in proportion, basophils were not different from the group control and that there was not a significant number of circulating activated basophils that could justify the allergic mediators release. The only patient with documented severe systemic mast cell 
mediator symptoms was no different from the other patients and controls.

Basophil activation test has been proposed as a reliable diagnostic test for in vitro diagnosis of IgE mediated reactions [24,27,28]. It is a complementary test used particularly when skin tests and allergenspecific IgE determinations cannot be performed or have given doubtful results in comparison with a patient's clinical history. To proper be performed it requires some particularities such as conservation of the blood samples, the preparation and quality of the reagents used, and the flow cytometry gating processes. The Flow-CAST test used to verify the different basophil activation stages include two markers: CD193 (CCR3) as a single basophil selection marker, reliable to identify peripheral blood basophils, and CD63 as an activation-related molecule [24].

The CD63 molecule it is most commonly basophil activationrelated marker used because of its commercial assay availability and also because its expression is closely associated with degranulation. As demonstrated in other studies the behavior of CD63 expression suggests that it appears on the cell surface during an anaphylactic degranulation by that it is expected a more intense up-regulation with a stronger trigger such as anti-FceRI.

Because of the clinical background of the patients, it was likely to observe a quicker and exuberant expression of CD63. However, in this study, we could not demonstrate differences between the control group, with no allergic background, and the patient group with a heavy allergic history.

As expected from previous studies [17], CD193 (CCR3) expression on basophils significantly decreased after stimulation with fMLP and anti-FceRI stimuli, in both mastocytosis patients and healthy individuals. However, no statistically significant differences were again registered between the 2 groups.

Th2 cells, which are known to express the CD294 (CRTH2) cell surface molecule, produce cytokines, such as IL-4, IL-5, IL-10 and IL-13, which play pivotal role in allergic diseases [18]. These cells can be analyzed by flow cytometry, allowing accurate and confident measurement of $\mathrm{T}$ cell frequency and surface phenotype. In the present study they were analyzed to corroborate the hypothesis of being outnumbered and over functional in patients with mastocytosis therefore, along with basophil and mast cells, be responsible for clinical manifestations.

When Th2 cells, were analyzed by flow cytometry the results were again disappointing, as significant differences were not observed between patients with mastocytosis and healthy individuals. In addition, the proportion of HLA-DR+ T cells in patients, were similar to that observed in controls.

In conclusion, it is well documented that mast cells have a central role in the pathogenesis of allergic reactions and, among patients studied and using the present methodology, the hypothesis that basophils and Th2 cells could be working together with mast cells, contributing to the clinical manifestations observed in patients with mastocytosis was poured down. Nevertheless, the small number of patients included must be taken into account and the hypothesis previously referred should be further investigated using different stimuli.

\section{Author Contributions}

All authors gave substantial contributions to conception and design of the study, acquisition, analysis and interpretation of data, drafting the article or revising it critically for important intellectual content, and they all gave the approval of the final version of the manuscript to be published. Individual contributions were especially relevant in the areas mentioned bellow.

Ana Spínola: literature review, study design, basophil immunophenotyping and basophil activation tests and data analysis, recording clinical data from patients with mastocytosis, statistical analysis and manuscript writing.

Joana Guimarães and Marlene Santos: basophil immunophenotyping and basophil activation tests.

Catarina Lau and Maria dos Anjos Teixeira: laboratorial diagnosis of mastocytosis, especially mast cell immunophenotyping data analysis and interpretation.

Rosário Alves: consulting and recording and interpreting clinical data from patients with mastocytosis.

Margarida Lima: literature review, study design, consulting and recording of clinical data from patients with mastocytosis, data analysis of mast cell immunophenotyping and laboratorial diagnosis of mastocytosis, data analysis of basophil immunophenotyping and basophil activation tests, and manuscript writing and revising it critically for important intellectual content.

\section{Acknowledgement}

The authors thank all persons who indirectly contributed to this work, namely to all the technicians from the Laboratory of Cytometry, as well as to the patients and blood donors that voluntary agreed to participate in this study.

\section{References}

1. Knol EF, Olszewski M (2011) Basophils and mast cells: Underdog in immune regulation? Immunol Lett 138: 28-31.

2. Brockow K, Metcalfe DD (2010) Mastocytosis. Chem Immunol Allergy 95: 110 124.

3. Worobec A, Metcalfe DD (2009) Wintrobe's Clinical Immunology. In: Woltzer Kluwer (Ed) Systemic Mastocytosis. (Edn), Lippincott Williams and Wilkins, Philadelphia, USA

4. Horny HP (2009) Mastocytosis: an unusual clonal disorder of bone marrowderived hematopoietic progenitor cells. Am J Clin Pathol 132: 438-447.

5. Horny HP Akin C, Metcalfe DD, Escribano L, Bennett JM, et al. (2008) Mastocytosis (Mast cell disease). In: Swerdlow, SH, Campo E, Harris NL, Jaffe ES, Pileri SA et al. (Eds) World Health Organization (WHO) Classification of Tumours. Pathology and Genetics. Tumours of Haematopoietic and Lymphoid Tissues. Lyon, France: IARC Press 2: 54-63

6. Valent P, Akin C, Arock M, Brockow K, Butterfield JH, et al. (2012) Definitions criteria and global classification of mast cell disorders with special reference to mast cell activation syndromes: a consensus proposal. Int Arch Allergy Immunol 157: 215-225

7. Brown JM, Wilson TM, Metcalfe DD (2008) The mast cell and allergic diseases: role in pathogenesis and implications for therapy. Clin Exp Allergy 38: 4-18.

8. Siracusa MC, Kim BS, Spergel JM, Artis D (2013) Basophils and allergic inflammation. J Allergy Clin Immunol 132: 789-801.

9. Han X, Jorgensen JL, Brahmandam A, Schlette E, Huh YO, et al. (2008) Immunophenotypic study of basophils by multiparameter flow cytometry. Arch Pathol Lab Med 132: 813-819.

10. Valent P (2010) Basophil Activation Antigens: Molecular Mechanisms and Clinical Implications. The Open Allergy Journal 3:52-59

11. Kostenis E, Ulven T (2006) Emerging roles of DP and CRTH2 in allergic inflammation. Trends Mol Med 12: 148-158.

12. Pettipher $R$ (2008) The roles of the prostaglandin $D(2)$ receptors $D P(1)$ and CRTH2 in promoting allergic responses. Br J Pharmacol 153 Suppl 1: S191199. 
Citation: Spínola A, Guimarães J, Santos M, Lau C, Teixeira MA, et al. (2014) Are Basophils and T helper 2 Cells Implicated in Mastocytosis? J Allergy Ther 5: 192. doi:10.4172/2155-6121.1000192

13. Knol EF, Mul FP, Jansen H, Calafat J, Roos D (1991) Monitoring human basophil activation via CD63 monoclonal antibody 435. J Allergy Clin Immuno 88: $328-338$

14. de Weck AL, Sanz ML (2003) For allergy diagnostic flow cytometry, detection of CD203c instead of CD63 is not at all an improvement in other hands. Clin Exp Allergy 33: 849-852.

15. Bühring HJ, Streble A, Valent $P$ (2004) The basophil-specific ectoenzyme E-NPP3 (CD203c) as a marker for cell activation and allergy diagnosis. Int Arch Allergy Immunol 133: 317-329.

16. Hennersdorf F, Florian S, Jakob A, Baumgärtner K, Sonneck K, et al. (2005) Identification of CD13, CD107a, and CD164 as novel basophil-activation markers and dissection of two response patterns in time kinetics of IgEdependent upregulation. Cell Res 15: 325-335.

17. Sainte-Laudy J, Ouk C (2010) Use of lipid rafting for the analysis of human basophil activation by flow cytometry. Inflamm Res 59 Suppl 2: S193-195.

18. Wambre E, James EA, Kwok WW (2012) Characterization of CD4+ T cel subsets in allergy. Curr Opin Immunol 24: 700-706.

19. Nagata K, Hirai H, Tanaka K, Ogawa K, Aso T, et al. (1999) CRTH2, an orphan receptor of T-helper-2-cells, is expressed on basophils and eosinophils and responds to mast cell-derived factor(s). FEBS Lett 459: 195-199.

20. Cosmi L, Annunziato F, Galli MIG, Maggi RME, Nagata K, et al. (2000) CRTH2 is the most reliable marker for the detection of circulating human type 2 Th and type 2 T cytotoxic cells in health and disease. Eur J Immunol 30: 2972-2979.
21. Cosmi L, Annunziato F, Galli G, Manetti R, Maggi E, et al. (2001) CRTH2 marker for the detection of human Th2 and Tc2 cells. Adv Exp Med Biol 495: 25-29.

22. Hirai H, Tanaka K, Yoshie O, Ogawa K, Kenmotsu K, et al. (2001) Prostaglandin D2 selectively induces chemotaxis in T helper type 2 cells, eosinophils, and basophils via seven-transmembrane receptor CRTH2. J Exp Med 193: 255261.

23. Xue L, Barrow A, Pettipher R (2009) Novel function of CRTH2 in preventing apoptosis of human Th2 cells through activation of the phosphatidylinositol 3-kinase pathway. J Immunol 182: 7580-7586.

24. Sanz ML, Maselli JP, Gamboa PM, Oehling A, Diéguez I, et al. (2002) Flow cytometric basophil activation test: a review. J Investig Allergol Clin Immuno 12: 143-154.

25. de Weck AL, Sanz ML, Gamboa PM, Aberer W, Bienvenu J et al. (2008) Diagnostic tests based on human basophils: more potentials and perspectives than pitfalls. Int J All Immunol 146: 177-189

26. Chirumbolo S, Vella A, Ortolani R, De Gironcoli M, Solero P, et al. (2008) Differential response of human basophil activation markers: a multi-parameter flow cytometry approach. Clin Mol Allergy 6: 12.

27. Ebo DG, Bridts CH, Hagendorens MM, Aerts NE, De Clerck LS, et al. (2008) Basophil activation test by flow cytometry: present and future applications in allergology. Cytometry B Clin Cytom 74: 201-210.

28. MacGlashan DW Jr (2013) Basophil activation testing. J Allergy Clin Immuno 132: $777-787$ 